\title{
Numerical solution through mathematical modelling of unsteady MHD flow past a semi- infinite vertical moving plate with chemical reaction and radiation
}

https://doi.org/10.2478/sgem-2018-0041

received August 27, 2018; accepted November 15, 2018.

\begin{abstract}
In the present manuscript, unsteady magnetohydrodynamic (MHD) flow over a moving porous semi-infinite vertical plate with time-dependent suction has been studied in the presence of chemical reaction and radiation parameters. Time-dependent partial differential equations in the dimensionless form are solved numerically through mathematical modelling in COMSOL Multiphysics. The results are obtained for velocity, temperature and concentration profiles at different times. Steady state results are also presented for different values of physical parameters. The parameters involved in the problem are useful to change the characteristics of velocity, heat transfer and concentration profiles. The numerical solution of partial differential equations involved in the problem is obtained without sacrificing the relevant physical phenomena.
\end{abstract}

Keywords: MHD flow; chemical reaction; radiation; numerical solution.

\section{Nomenclature}

$\begin{array}{ll}x^{\prime} & \text { Axial coordinate } \\ y^{\prime} & \text { Transverse coordinate } \\ y & \text { Dimensionless distance to the surface } \\ t^{\prime} & \text { Time } \\ t & \text { Dimensionless time } \\ u^{\prime} & \text { Velocity component in } x^{\prime} \text { direction } \\ v^{\prime} & \text { Velocity component in } y^{\prime} \text { direction } \\ u & \text { Dimensionless axial velocity }\end{array}$

*Corresponding author: Anupam Bhandari, Department of Mathematics, School of Engineering , University of Petroleum \& Energy Studies (UPES), Energy Acres Building, Bidholi, Dehradun 248007, Uttarakhand, India, E-mail: pankaj.anupam6@gmail.com $u_{p}^{\prime} \quad$ Wall velocity

$u_{p} \quad$ Dimensionless wall velocity

$V_{0} \quad$ Scale for suction velocity

$C^{\prime}$ Concentration

$C_{w}$ Wall concentration

$C_{\infty} \quad$ Concentration away from the wall

$C$ Dimensionless concentration

$T^{\prime} \quad$ Temperature

$T_{w} \quad$ Wall temperature

$T_{\infty} \quad$ Free stream temperature

$\theta$ Dimensionless temperature

$K_{1} \quad$ Chemical reaction parameter

$K^{\prime} \quad$ Permeability of porous medium

$K \quad$ Dimensionless permeability parameter

$B_{0} \quad$ Magnetic induction

$D$ Mass diffusion coefficient

$g \quad$ Acceleration due to gravity

$Q_{0} \quad$ Heat absorption coefficient

$C_{p} \quad$ Specific heat at constant pressure

$Q_{1}^{\prime} \quad$ Radiation absorption coefficient

$Q_{1} \quad$ Dimensionless absorption coefficient

$\varphi \quad$ Dimensionless heat generation/absorption parameter

$\gamma \quad$ Dimensionless chemical reaction parameter

$\mathrm{Pr}$ Prandtl number

Sc Schmidt number

$G r$ Thermal Grashof number

Gm Solutal Grashof number

$\mu \quad$ Dynamic viscosity

$\rho$ Density

$\omega$ Very small numerical number

$F(t)$ Function of $t$

$\varphi_{1} \quad$ Volume fraction

$m$ Effective magnetic number 


\section{Introduction}

Magnetohydrodynamics (MHD) is concerned with the study of the interaction between magnetic fields and fluid conductors of electricity. The body force acting on the fluid is the Lorentz force that arises when electric current flows at an angle to the direction of an impressed magnetic field. Due to increasing applications of MHD, researchers have been attracted to study the various types of flow to analyse the velocity, temperature and concentration profiles for different dimensionless parameters. Most of the research papers on MHD flow are basically focussed on the analytic solution; however, the accuracy of the analytic solution is very weak. Even though a number of computational tools are available to solve non-linear coupled partial differential equations, accuracy is the problem with the computational tools. To find a solution with the desired degree of accuracy, mathematical modelling has become an important tool currently. The present problem has also been modelled in COMSOL Multiphysics, and the solutions of the partial differential equations have been obtained without sacrificing the relevant physical phenomena.

The effects of slip condition on an unsteady electrically conducting MHD flow past a periodically accelerated horizontal porous plate under the influence of transverse magnetic field and the Hall current have recently been investigated [1]. The effects of optical properties of radiation and the Lorentz force on an unsteady laminar boundary MHD fluid flow and heat transfer along a semi-infinite stretching plate have been numerically investigated [2]. Unsteady squeezing MHD nanofluid flow and heat transfer in the presence of thermal radiation impact and considering the Cattaneo-Christov heat flux model, instead of the conventional Fourier's law of heat conduction, have also been examined [3]. The effects of temperature-dependent viscosity on the MHD boundary layer flow past a vertical porous plate have been studied in the presence of a magnetic field, thermal radiation, thermophoresis through heat and mass transfer [4]. Heat transfer analysis on MHD nanofluids in a porous channel with stretching walls has been studied [5]. The laminar boundary layer flow driving past a semi-infinite flat plate has been studied taking into account the effects of a magnetic field [6].

The unsteady MHD flow of a Brinkman type nanofluid over a vertical plate embedded in a porous medium with variable surface velocity, temperature and concentration has been investigated [7]. The chemical reaction effect on a boundary layer flow of an electrically conducting micropolar fluid subject to a transverse magnetic field along a vertical plate with variable wall temperature and concentration has been reported [8]. MHD mixed convection and mass transfer flow over a semi-infinite porous inclined plate in the presence of thermal radiation with variable suction and thermophoresis has been analysed numerically [9].

The effect of chemical reactions on an unsteady MHD flow past a semi-infinite vertical porous plate with viscous dissipation has been previously analysed [10]. Analytical solutions for electrically conducting and heat generating/ absorbing fluid on a continuously vertical permeable surface in the presence of radiation, a first-order homogeneous chemical reaction and mass flux have been reported [11]. The motion of an electrically conducting fluid in the presence of an external magnetic field has been investigated [12]. The heat generation/absorption and thermo-diffusion of an unsteady free convective MHD flow of a radiating and chemically reactive fluid near an infinite vertical plate through a porous medium with Hall current have been studied [13].

An electrically conducting fluid flow in an array of square ducts, separated by conducting walls of arbitrary thickness, subject to an applied magnetic field has been investigated [14]. An investigation of unsteady hydromagnetic natural convection heat and mass transfer flow past an impulsively moving infinite vertical plate embedded in a uniform porous medium when the temperature of the plate has a temporarily ramped profile has been carried out [15]. The effects of a magnetic field on the three-dimensional flow of a nanofluid having a suspension of ferrous nanoparticles within the framework of a non-uniformly thickened sheet in a slip flow regime has been analysed [16]. An analytical model of a stationary hypersonic MHD shock with an externally applied magnetic field has also been proposed [17]. Decaying homogeneous and isotropic MHD turbulence has been investigated numerically at large Reynolds numbers [18]. A model of a laminar viscous conducting flow, near a dielectric disc in a uniform magnetic field and in the presence of external rotation, has been previously considered [19]. Nanofluid flow due to a rotating disc with variable thickness has been studied with heat and mass transfer subject to volume fraction of nanoparticle and homogeneousheterogeneous reactions [20]. The convection flow of a viscous fluid over a curved stretching sheet with heat and mass transfer has been reported [21]. Nanofluid flow on an exponentially radiating stretching sheet with heat and mass transfer has also been investigated [22]. Analysis of the boundary layer flow of a nanofluid over a stretching surface has been investigated in the presence of an applied magnetic field and a chemical reaction [23]. 


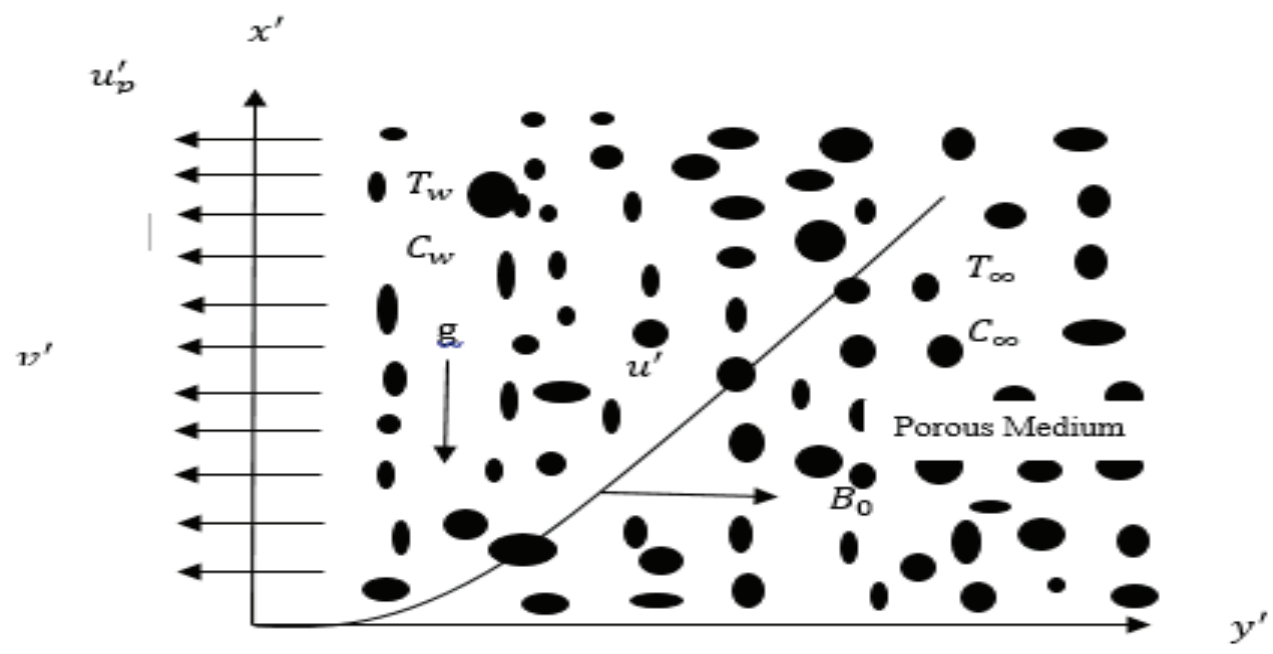

Figure 1: Sketch of the flow geometry.

In the present work, two-dimensional unsteady laminar flow and MHD free convection with heat and mass transfer is investigated. The viscosity of the magnetic fluid depends on the magnetic field and the fluid is incompressible. It passes through a semi-infinite vertical porous moving plate in a porous medium. The magnetic field is subjected to a transverse direction in the presence of thermal buoyancy, concentration buoyancy and radiation effects in the fluid. The geometry of the flow is given in Fig.1.

\section{Mathematical formulation}

The governing equations for the problem are as follows:

$$
\begin{gathered}
\frac{\partial v^{\prime}}{\partial y^{\prime}}=0 \\
\frac{\partial u^{\prime}}{\partial t^{\prime}}+v^{\prime} \frac{\partial u^{\prime}}{\partial y^{\prime}}=g \beta\left(T^{\prime}-T_{\infty}\right)+g \beta^{\prime}\left(C^{\prime}-C_{\infty}\right)+ \\
+\frac{\mu}{\rho}\left(1+\frac{3}{2} \varphi_{1} m\right) \frac{\partial^{2} u^{\prime}}{\partial y^{\prime 2}}-\frac{\sigma}{\rho} B_{0}^{2} u^{\prime}-u^{\prime} \frac{\mu}{\rho K^{\prime}} \\
\frac{\partial T^{\prime}}{\partial t^{\prime}}+v^{\prime} \frac{\partial T^{\prime}}{\partial y^{\prime}}=\frac{k}{\rho C_{p}} \frac{\partial^{2} T^{\prime}}{\partial y^{\prime 2}}-\frac{Q_{0}}{\rho C_{p}}\left(T^{\prime}-T_{\infty}\right)+Q_{1}^{\prime}\left(C^{\prime}-C_{\infty}\right)
\end{gathered}
$$

$$
\frac{\partial C^{\prime}}{\partial t^{\prime}}+v^{\prime} \frac{\partial C^{\prime}}{\partial y^{\prime}}=D \frac{\partial^{2} C^{\prime}}{\partial y^{\prime 2}}-K_{1}\left(C^{\prime}-C_{\infty}\right)
$$

In Eq. (2) (Momentum equation), the expressions $g \beta\left(T^{\prime}-T_{\infty}\right)$ and $g \beta^{\prime}\left(C^{\prime}-C_{\infty}\right)$ indicate the thermal and concentration buoyancy effects, respectively. In Eq. (3) (Temperature equation), the expressions $\frac{Q_{0}}{\rho C_{p}}\left(T^{\prime}-T_{\infty}\right)$ and $Q_{1}^{\prime}\left(C^{\prime}-C_{\infty}\right)$ denote the heat absorption and radiation effects, respectively. In Eq. (4) (Concentration equation), the expression $K_{1}\left(C^{\prime}-C_{\infty}\right)$ represents the chemical concentration buoyancy effects. In this problem, it is supposed that the permeable plate moves with a constant velocity in the direction of magnetic fluid flow. It is also assumed that the temperature, concentration and the suction velocity are functions of time. For such a type of flow, the boundary conditions are as follows:

$$
\begin{gathered}
u^{\prime}=u_{p}^{\prime}, \quad T^{\prime}=T_{w}, \quad C^{\prime}=C_{w} \quad \text { at } \quad y^{\prime}=0 \\
u^{\prime} \rightarrow U^{\prime}\left(t^{\prime}\right), \quad T^{\prime} \rightarrow T_{\infty}, C^{\prime} \rightarrow C_{\infty} \text { at } y^{\prime} \rightarrow \infty
\end{gathered}
$$

From Eq. (1), it is clear that the suction velocity is normal to the plate and it is only a function of time. It can be taken in exponential form as follows:

$$
v^{\prime}=-V_{0}(1+\omega F(t))
$$

The above equations can be made dimensionless with the help of the following dimensionless quantities:

$$
\begin{gathered}
u=\frac{u^{\prime}}{V_{0}}, v=\frac{v^{\prime}}{V_{0}}, y=\frac{\rho V_{0} y^{\prime}}{\mu}, u_{p}=\frac{u_{p}^{\prime}}{V_{0}}, \\
\theta=\frac{T^{\prime}-T_{\infty}}{T_{w}-T_{\infty}}, C=\frac{C^{\prime}-C_{\infty}}{C_{w}-C_{\infty}}
\end{gathered}
$$




$$
\begin{gathered}
U(t)=\frac{u^{\prime}\left(t^{\prime}\right)}{V_{0}}, t=\frac{\rho V_{0}^{2}}{\mu} t^{\prime}, G r=\frac{\mu g \beta\left(T_{w}-T_{\infty}\right)}{\rho V_{0}^{3}}, \\
G m=\frac{\mu g \beta^{\prime}\left(C_{w}-C_{\infty}\right)}{\rho V_{0}^{3}} \\
M=\frac{\sigma B_{0}^{2} \mu}{\rho^{2} V_{0}^{2}}, K=\frac{K^{\prime} V_{0}^{2} \rho^{2}}{\mu^{2}}, \gamma=\frac{K_{1} \mu}{\rho V_{0}^{2}}, S c=\frac{\mu}{\rho D}, \\
\varphi=\frac{Q_{0} \mu}{\rho^{2} C_{p} V_{0}^{2}}, \operatorname{Pr}=\frac{\mu C_{p}}{k}, Q_{1}=\frac{\mu Q_{1}^{\prime}\left(C_{w}-C_{\infty}\right)}{\rho V_{0}^{2}\left(T_{w}-T_{\infty}\right)}
\end{gathered}
$$

Using the above dimensionless relations, Eqs. (2)-(4) can be written as follows:

$$
\begin{gathered}
\frac{\partial u}{\partial t}-(1+\omega F(t)) \frac{\partial u}{\partial y}-\left(1+\frac{3}{2} \varphi_{1} m\right) \frac{\partial^{2} u}{\partial y^{2}}+\left(M+\frac{1}{K}\right) u-G r \theta-G m C=0 \\
\frac{\partial \theta}{\partial t}-(1+\omega F(t)) \frac{\partial \theta}{\partial y}-\frac{1}{P r} \frac{\partial^{2} \theta}{\partial y^{2}}+\varphi \theta-Q_{1} C=0 \\
\frac{\partial C}{\partial t}-(1+\omega F(t)) \frac{\partial C}{\partial y}-\frac{1}{S c} \frac{\partial^{2} C}{\partial y^{2}}+\gamma C=0
\end{gathered}
$$

The boundary conditions for Eqs. (11)-(13) are as follows:

$$
\begin{gathered}
u=u_{p}, \theta=1, C=1 \text { at } y=0 \\
u \rightarrow U(t), \theta \rightarrow 0, C \rightarrow 0 \text { at } y \rightarrow \infty
\end{gathered}
$$

\section{Mathematical modelling and numerical solution}

Eqs. (11)-(13) are modelled in COMSOL Multiphysics. Here, a 1D time-dependent model is selected under the option model wizard and then the geometry is drawn. Then, all the parameters are inserted under the option "global definition". This modelling is done in the dimensionless mode. The above equations are modelled in COMSOL with the help of the following standard form of differential equation:

$$
e_{a} \frac{\partial^{2} \boldsymbol{u}}{\partial t^{2}}+d_{a} \frac{\partial \boldsymbol{u}}{\partial t}+\nabla \cdot(-c \nabla \hat{\boldsymbol{a}}-\boldsymbol{\alpha} \boldsymbol{u}+\boldsymbol{y})+. \nabla+\mathrm{a}=f
$$

where

$$
u=[u, \text { theta }, C]^{T} \text { and } \nabla=\frac{\partial}{\partial y}
$$

In Eq. (16), $c$ is called the diffusion coefficient, a is called the absorption coefficient, $f$ is called the source term, $e_{a}$ is called the mass coefficient, $d_{a}$ is called the damping coefficient, $\alpha$ is called the conservative flux convection coefficient, $\hat{\boldsymbol{a}}$ is called the convection coefficient and $\gamma$ is called the conservative flux source. For the boundary conditions, Dirichlet boundary conditions are selected and, for getting a more accurate solution, an extrafine mesh is selected. This type of modelling solves the problem through the finite element method. The element size is taken as 0.0001 . Finally, using a parametric sweep, the velocity, temperature and concentration profiles are obtained for different parameters and for different times.

\section{Results and discussion}

The present experiment was conducted to know the physical significance of the different parameters of the flow. Figs. 2, 3 and 4 are the unsteady results of the velocity, temperature and concentration profiles. However, Figs. 5-13 are the steady state results. Previously, researchers [11-12] have used the analytical methods to solve the Eqs of the type (11)-(13) including both steady and unsteady nature problems. However, real physical significance of the flow should be studied through unsteady state problems of MHD flow. The numerical results are obtained here through mathematical modelling in COMSOL without sacrificing the relevant physical phenomena of MHD flow.

\subsection{Unsteady state results}

Fig. 2 represents the velocity profile for different values of time. In the problem, the plate is moving and the magnetic field is applied in the transverse direction. The Reynolds number is taken to be very small to keep the flow laminar. It is clear from Fig. 2 that on increasing the values of time, the velocity decreases. At $t=0.25$, there is an effect of the moving plate on the velocity and, after certain time, its effects decrease, resulting in the velocity of the fluid decreasing.

Fig. 3 indicates the temperature profiles for different values of time. Due to MHD free convection in the fluid, heat is transferred through fluid flow. For increasing values of time, the temperatures in the flow also decrease. Comparing Figs. 2 and 3, it is observed that from $y=1$ to $y=3$, the decrease in the velocity in Fig. 2 is very less as compared to that at other points. Therefore, within this range, the heat transfer also is less than that at other points. 


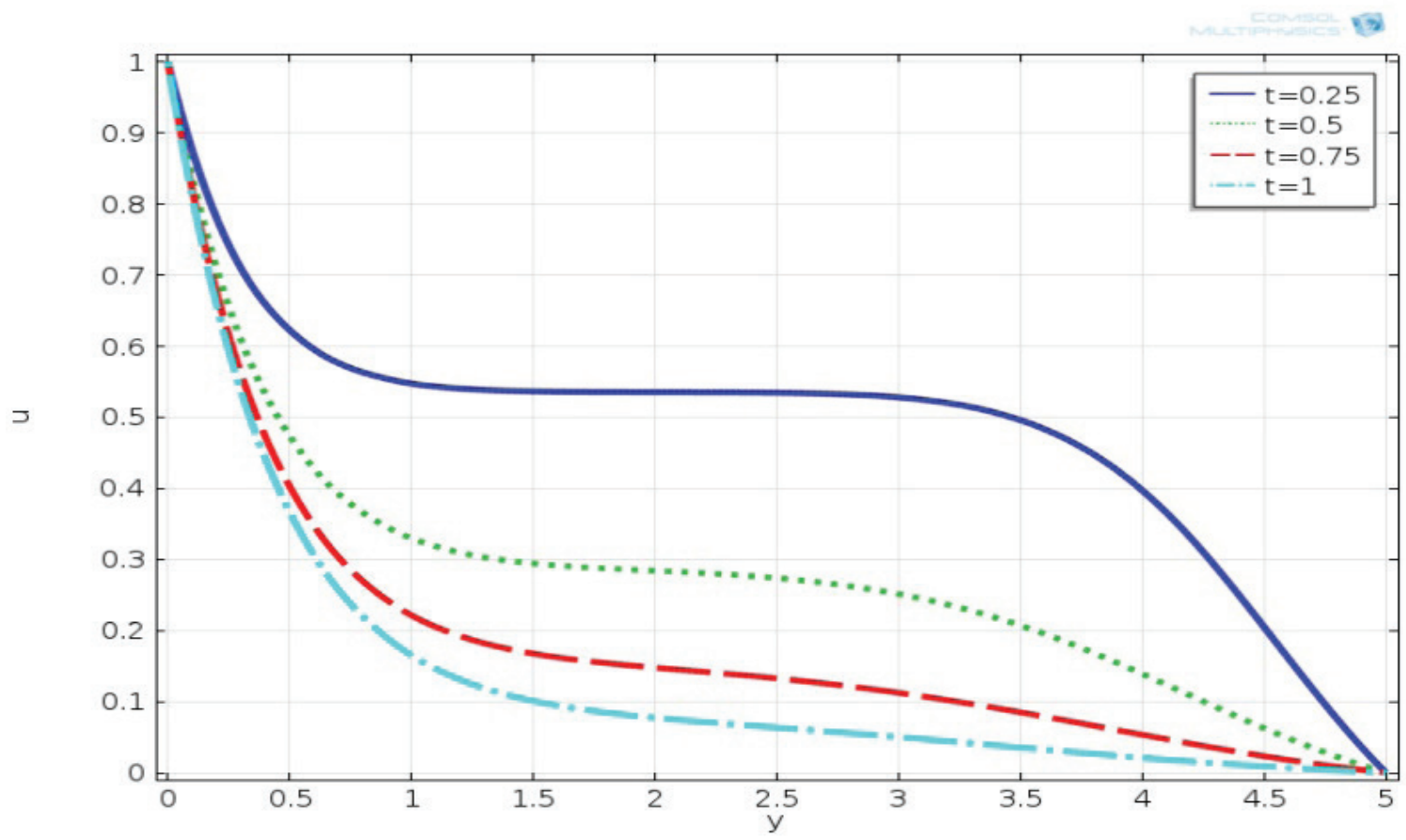

Figure 2: Velocity profiles for different times at $M=2, K=2, G r=2, G m=2, \operatorname{Pr}=0.72, \varphi=2, Q_{1}=2, S c=0.2, \gamma=0.2, \varphi_{1}=0.2, m=0.5$.

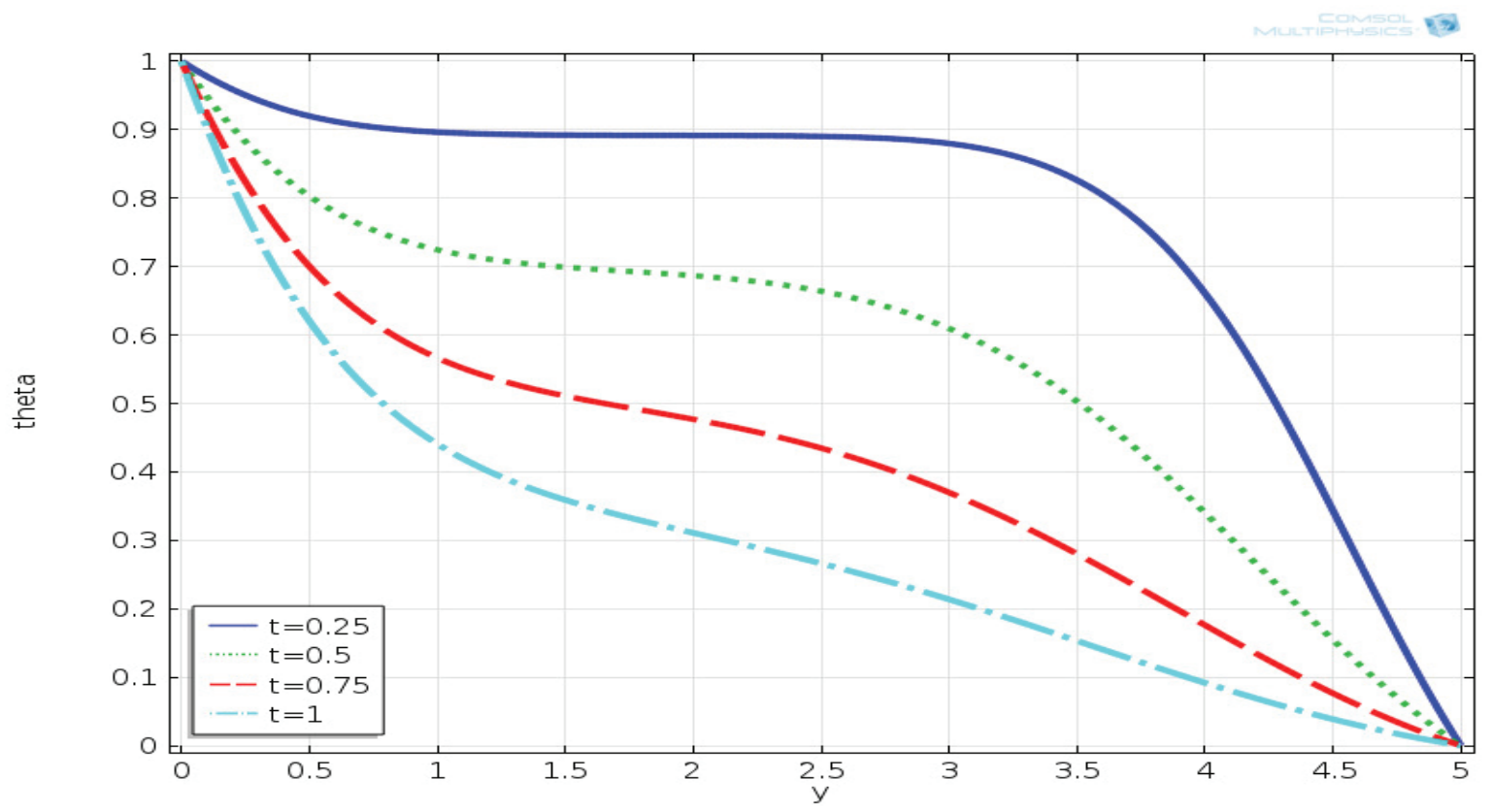

Figure 3: Temperature profiles for different times at $M=2, K=2, G r=2, G m=2, \operatorname{Pr}=0.72, \varphi=2, Q_{1}=2, S c=0.2, \gamma=0.2, \varphi_{1}=0.2, m=0.5$. 


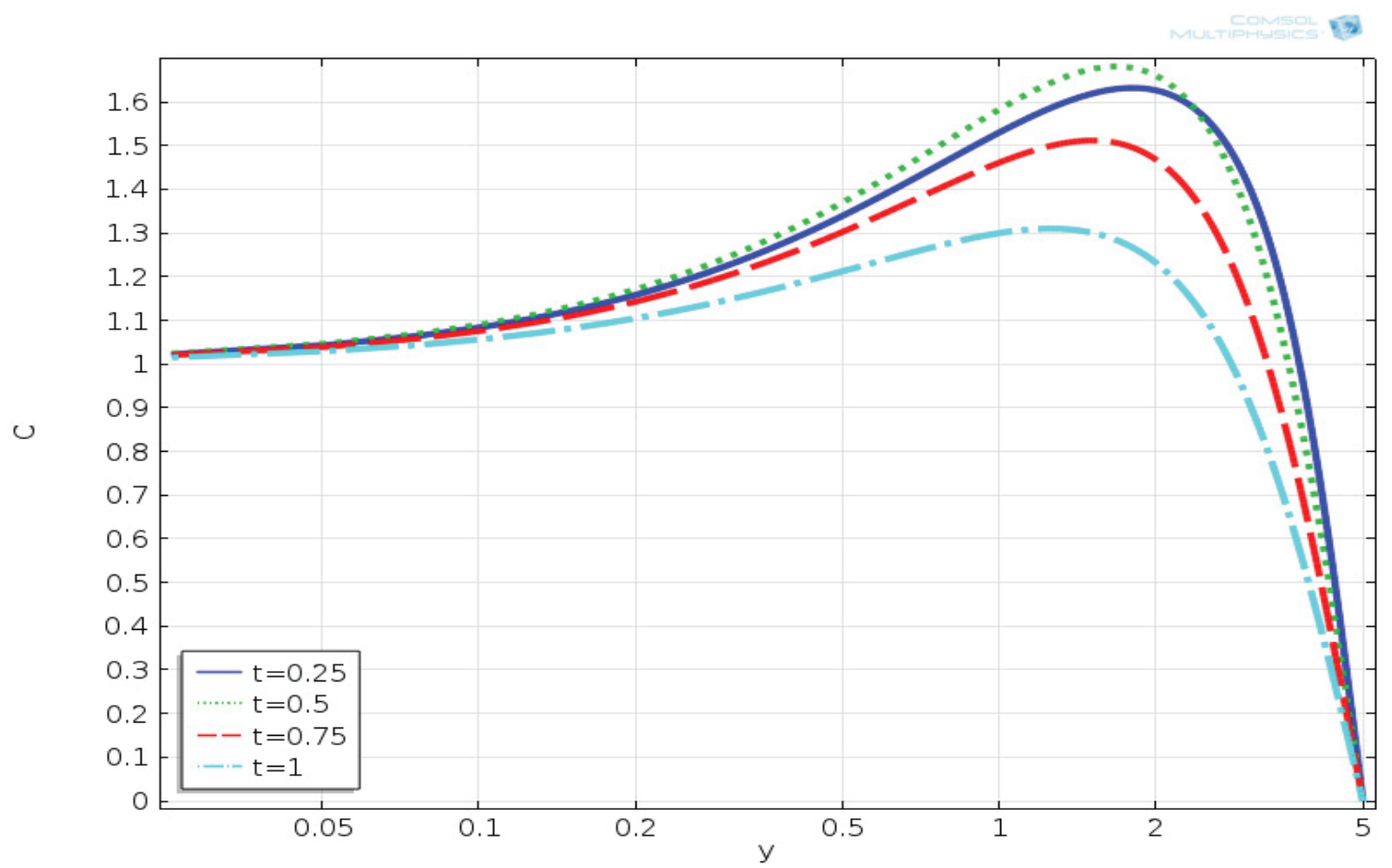

Figure 4: Concentration profiles for different times at $M=2, K=2, G r=2, G m=2, P r=0.72, \varphi=2, Q_{1}=2, S c=0.2, \gamma=0.2, \varphi_{1}=0.2, m=0.5$.

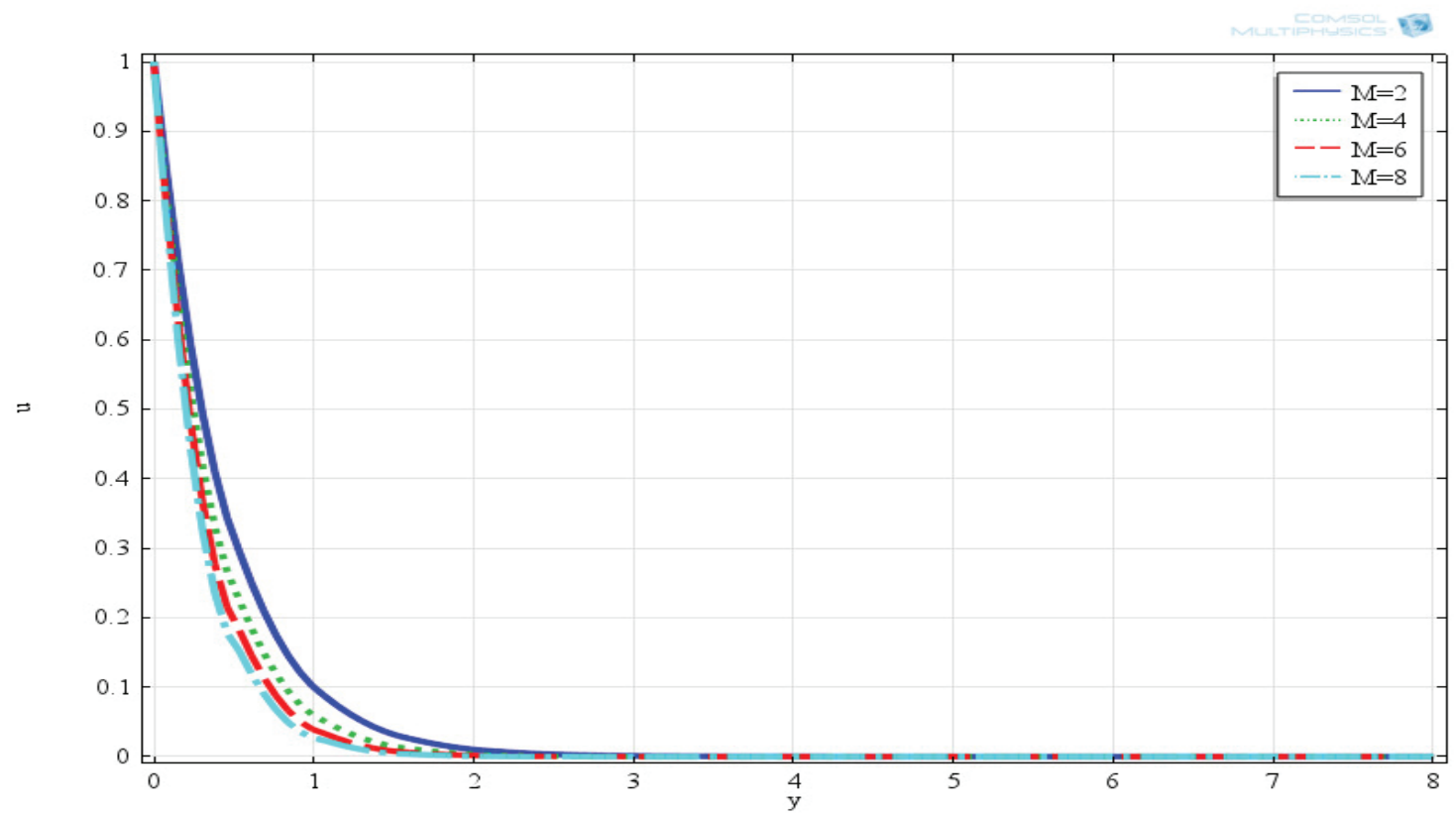

Figure 5: Steady state velocity profiles for different values of $M$ at $K=2, G r=2, G m=2, \operatorname{Pr}=0.72, \varphi=2, Q_{1}=2, S c=0.2, \gamma=0.2, \varphi_{1}=0.2, m=0.5$. 


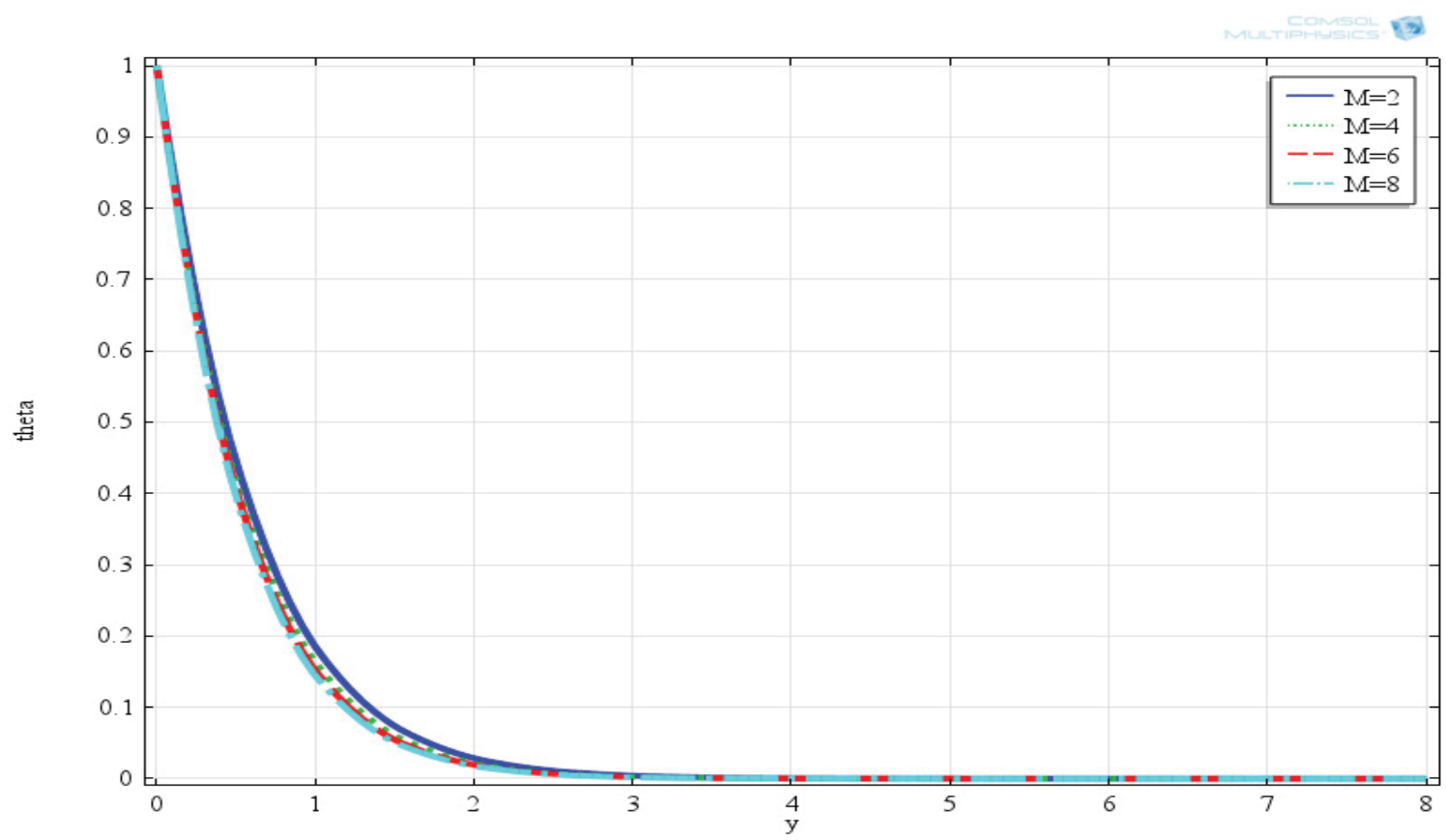

Figure 6: Steady state temperature profiles for different values of $M$ at $K=2, G r=2, G m=2, P r=0.72, \varphi=2, Q_{1}=2, S c=0.2, \gamma=0.2, \varphi_{1}=0.2, m=0.5$.

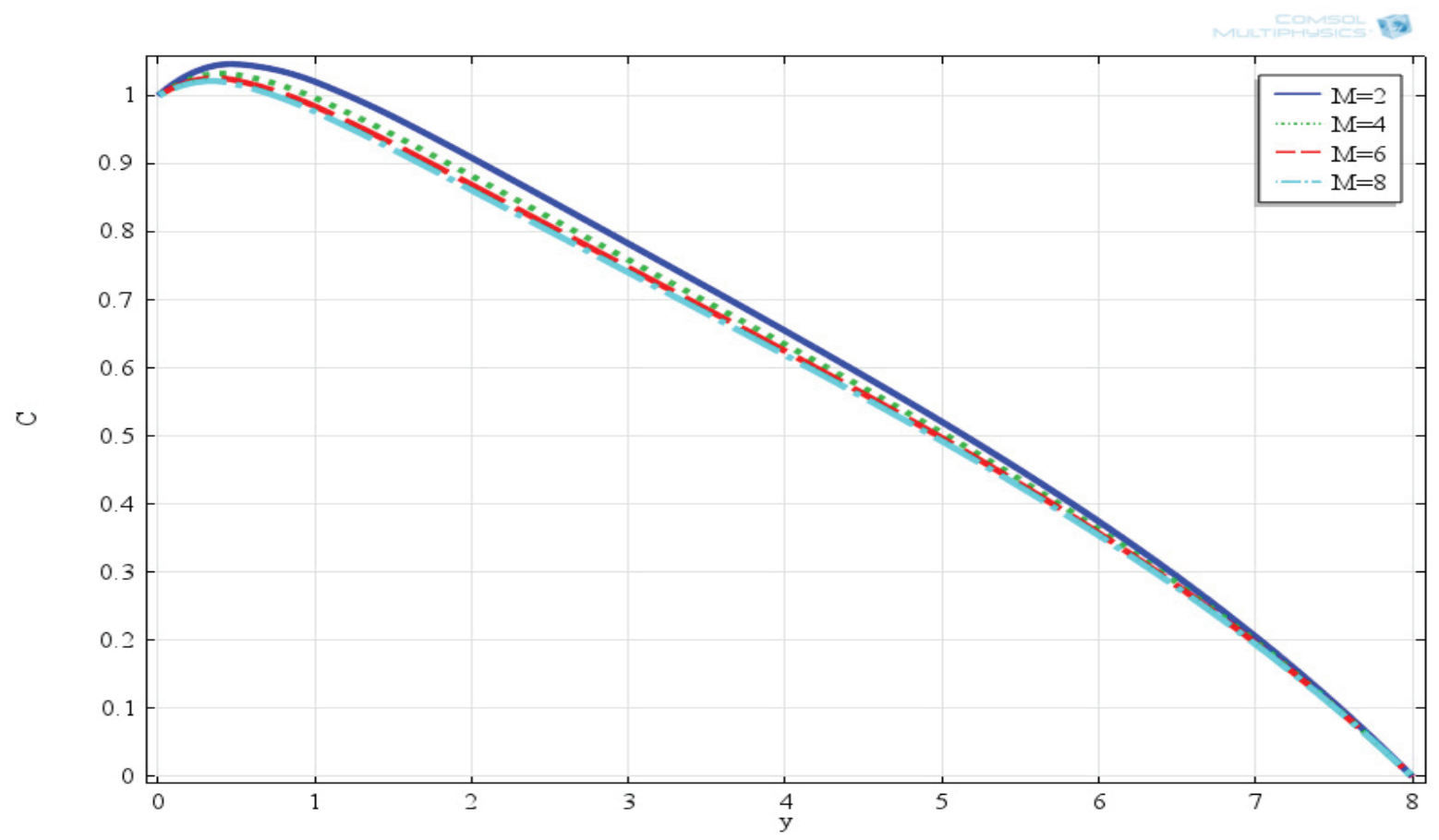

Figure 7: Steady state concentration profiles for different values of $M$ at $K=2, G r=2, G m=2, P r=0.72, \varphi=2, Q_{1}=2, S c=0.2, \gamma=0.2, \varphi_{1}=0.2, m=0.5$. 


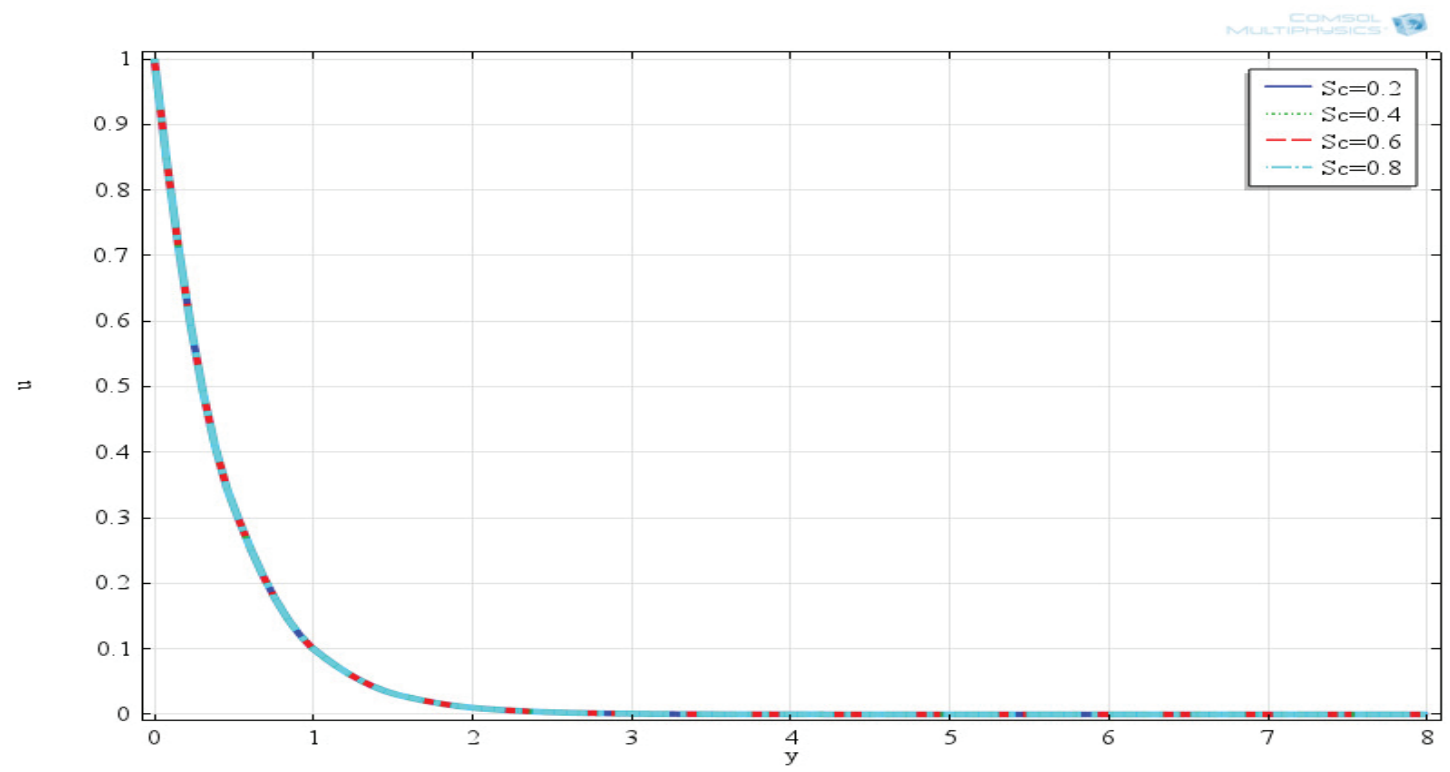

Figure 8: Steady state velocity profiles for different values of $S c$ at $M=2, K=2, G r=2, G m=2, \operatorname{Pr}=0.72, \varphi=2, Q_{1}=2, \gamma=0.2, \varphi_{1}=0.2, m=0.5$.

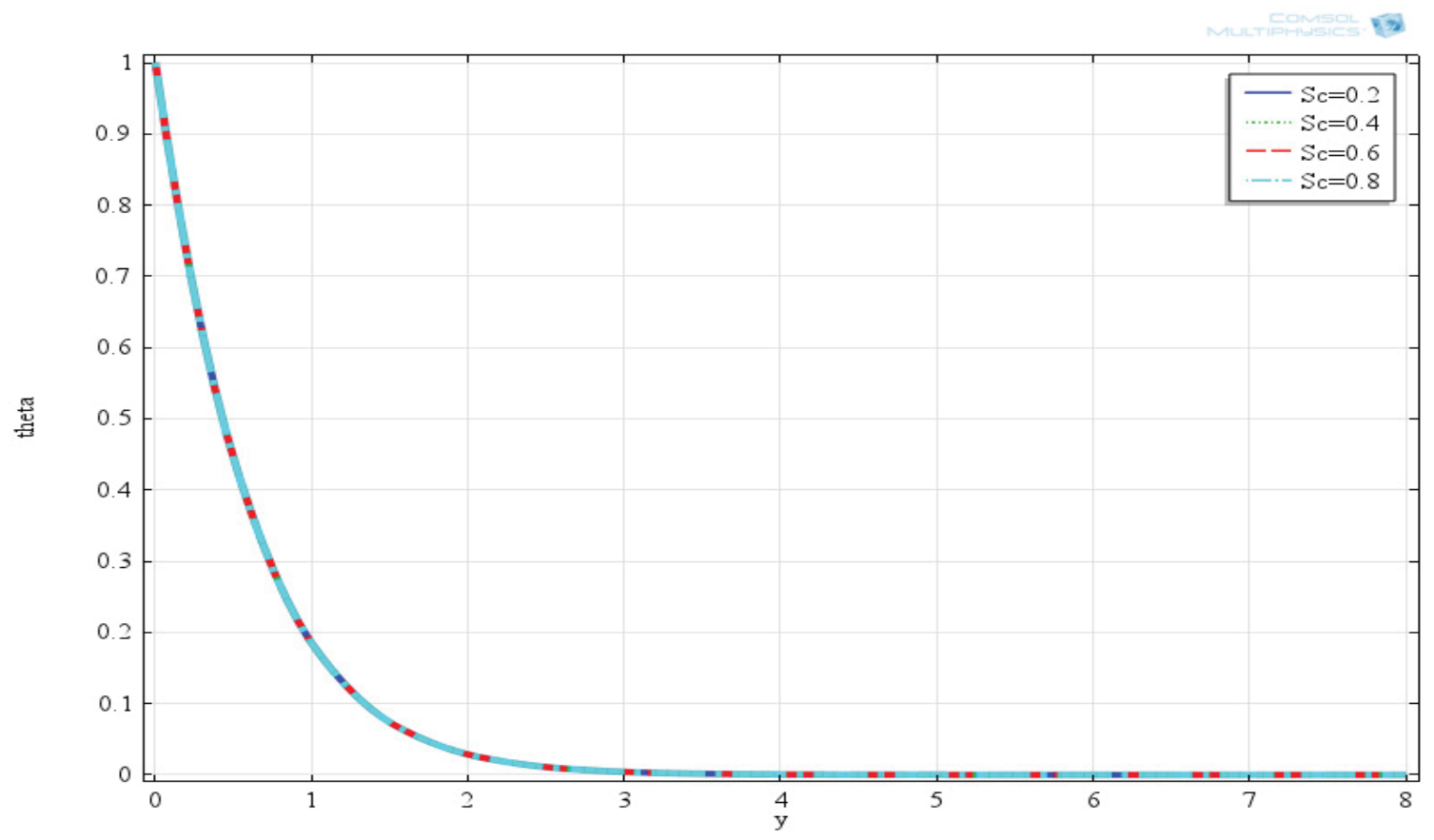

Figure 9: Steady state temperature profiles for different values of $S c$ at $M=2, K=2, G r=2, G m=2, \operatorname{Pr}=0.72, \varphi=2, Q_{1}=2, \gamma=0.2, \varphi_{1}=0.2, m=0.5$. 


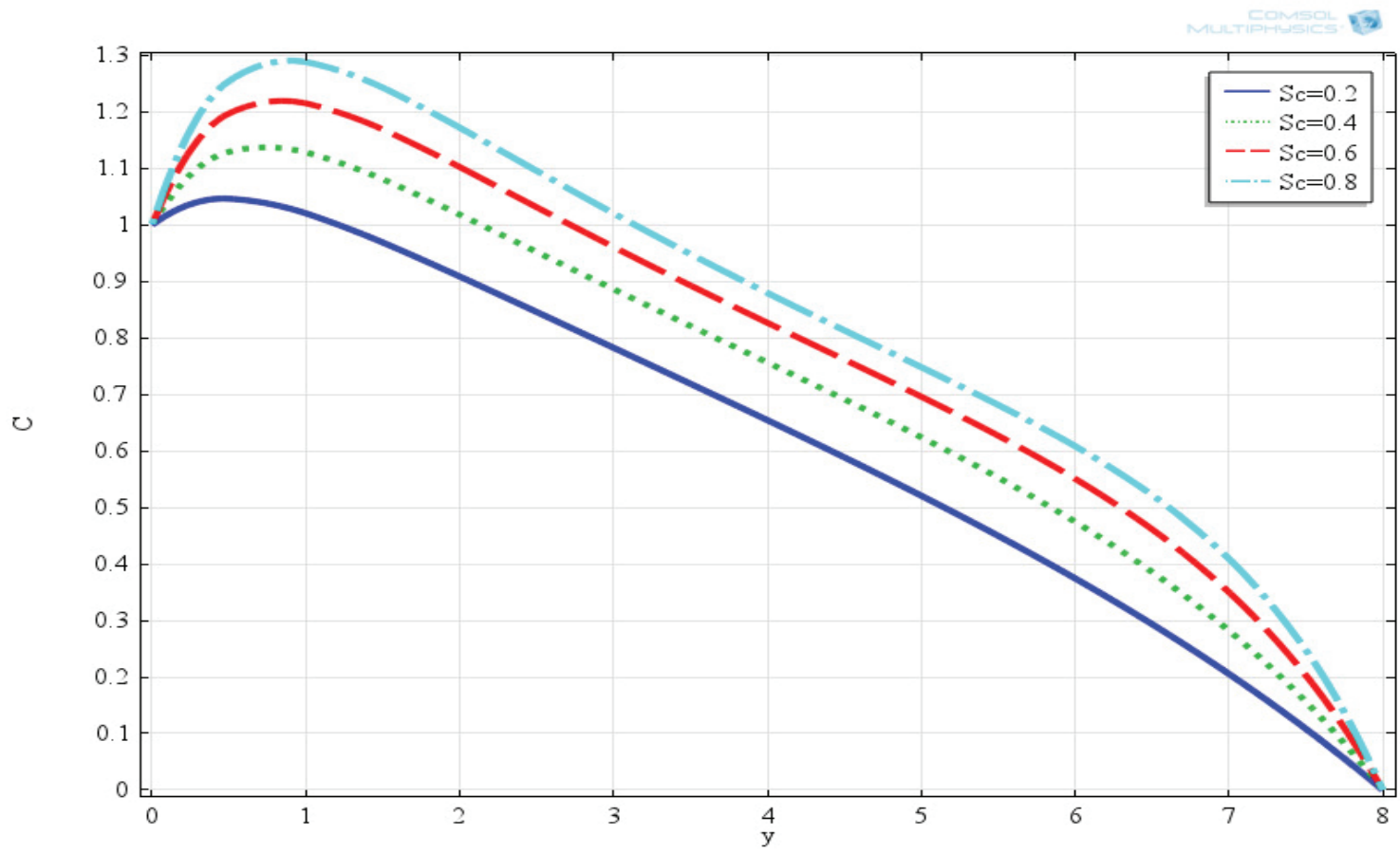

Figure 10: Steady state concentration profiles for different values of $S c$ at $M=2, K=2, G r=2, G m=2, P r=0.72, \varphi=2, Q_{1}=2, \gamma=0.2, \varphi_{1}=0.2, m=0.5$.

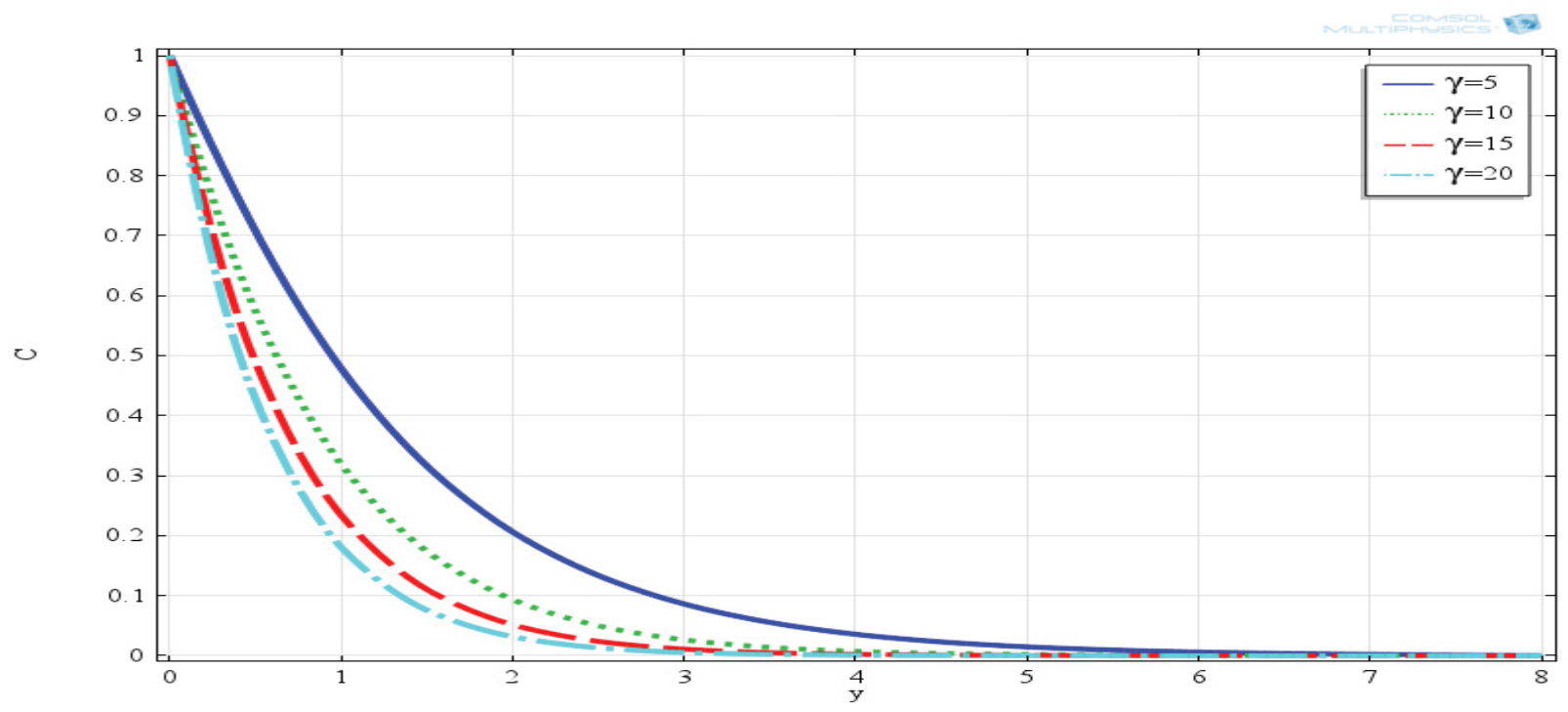

Figure 11: Steady state concentration profiles for different values of $\gamma$ at $M=2, K=2, G r=2, G m=2, \operatorname{Pr}=0.72, \varphi=2, Q_{1}=2, S c=0.2, \varphi_{1}=0.2, m=0.5$. 


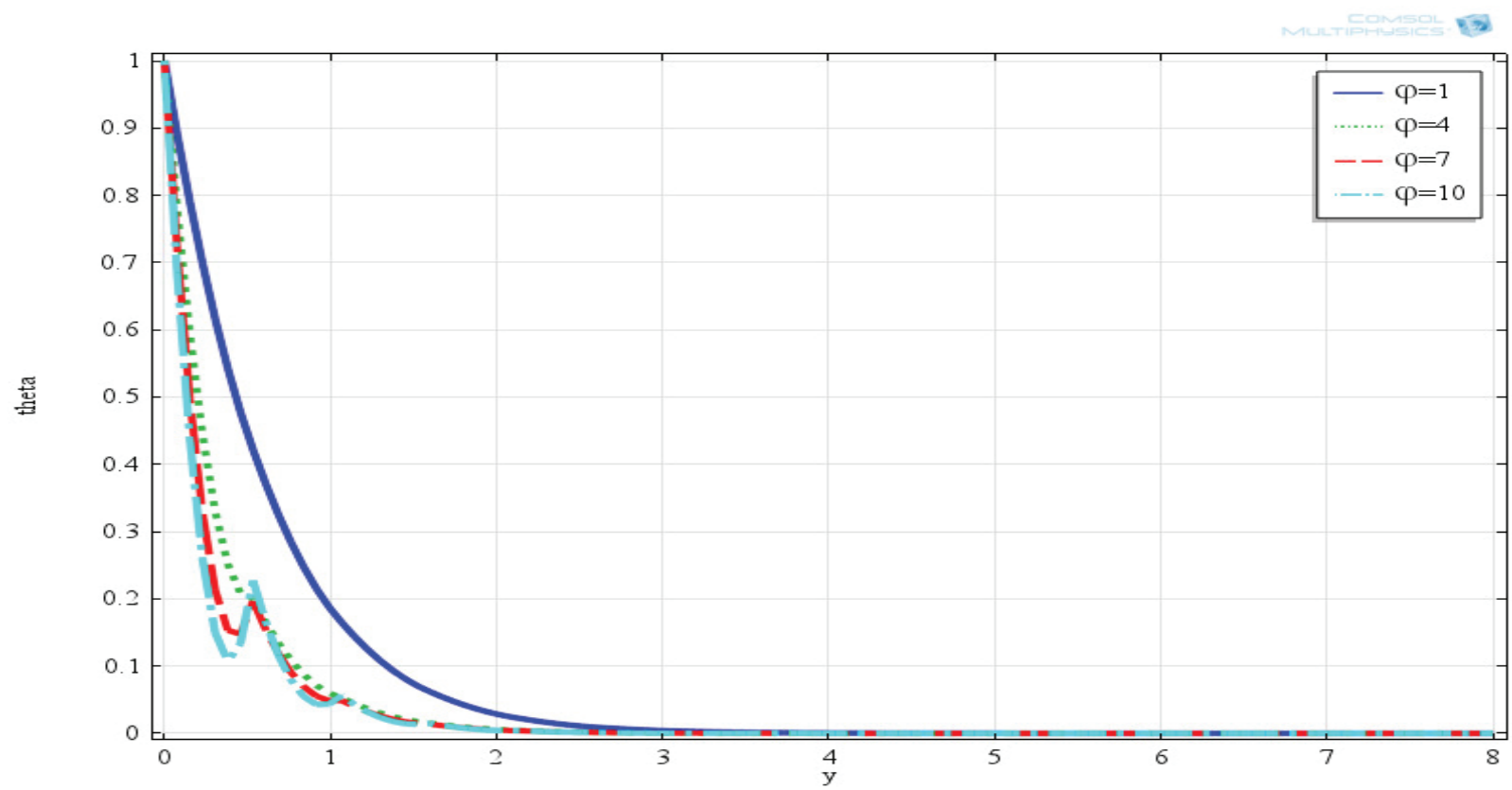

Figure 12: Steady state temperature profiles for different values of $\varphi$ at $M=2, K=2, G r=2, G m=2, \operatorname{Pr}=0.72, \gamma=0.2, Q_{1}=2, S c=0.2, \varphi_{1}=0.2, m=0.5$.

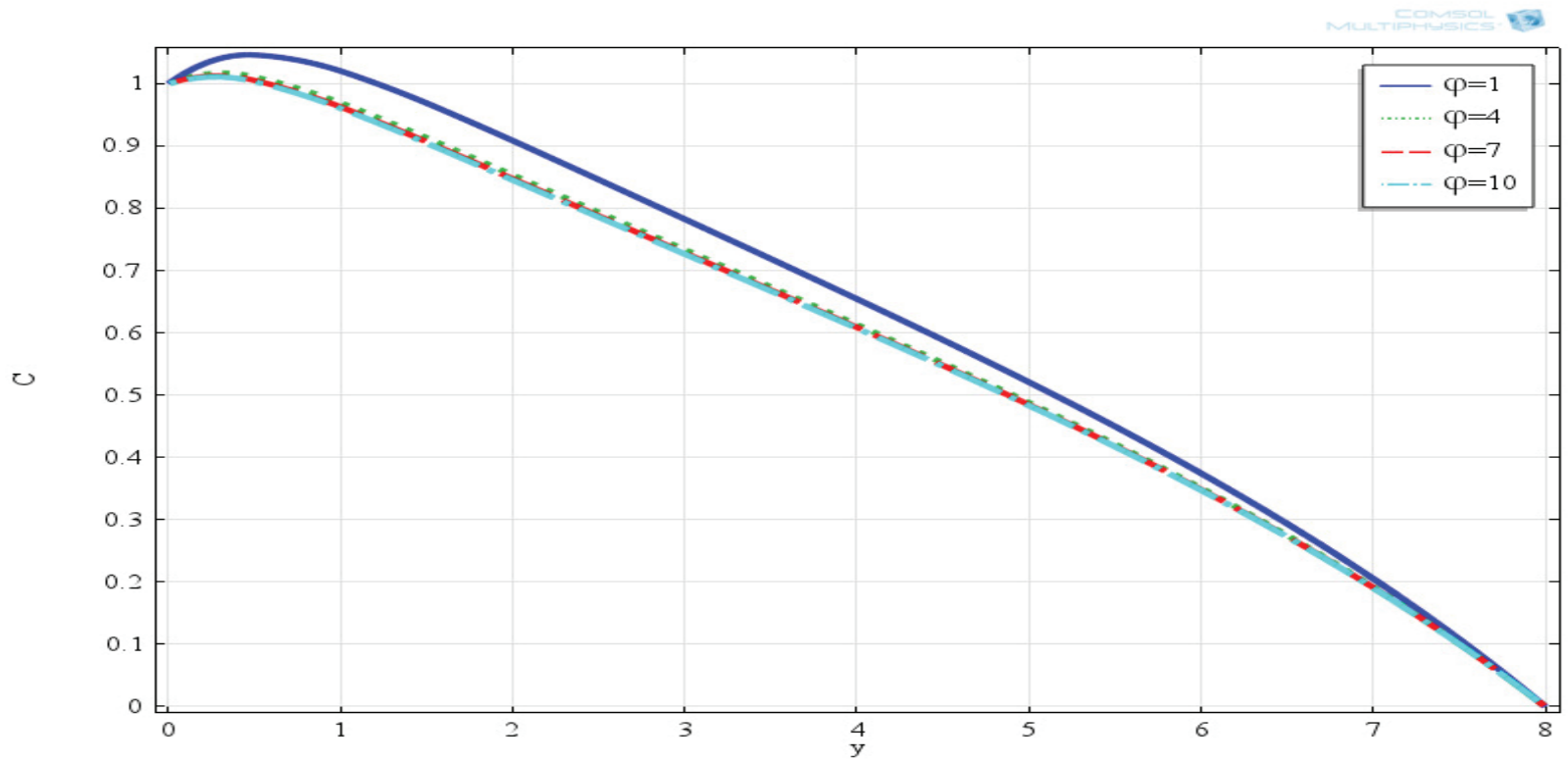

Figure 13: Steady state concentration profiles for different values of $\varphi$ at $M=2, K=2, G r=2, G m=2, P r=0.72, \gamma=0.2, Q_{1}=2, S c=0.2, \varphi_{1}=0.2, m=0.5$. 
Fig. 4 depicts the concentration profiles. Initially, for increasing values of time, the concentration remains approximately the same. However, it is clear from the results that the concentration increases between $y=1$ and $y=3$. It is observed that in this region, temperature and velocity decrease less than at other points. This result indicates that during the stable regions of velocity and temperature, the effect of concentration at different times can be seen clearly in Fig.4.

\subsection{Steady state results}

Fig. 5 shows the velocity profiles for different values of the magnetic parameter. Increasing the magnetic parameter, the velocity decreases here, but the magnetic parameter does not have a very large impact since the electric field is absent. Fig. 6 represents the temperature distribution for different values of the magnetic parameter. Increasing the magnetic parameter decreases the heat transfer in the fluid. The effect of the magnetic parameter on the concentration profile can also be observed clearly in Fig. 7. Decreasing heat transfer also decreases the concentration.

Figs. 8 and 9 represent the velocity and temperature profiles, respectively. Increasing the values of the parameter $S c$ does not have any impact on the velocity and temperature. However, it has a significant impact on concentration profile, as seen in Fig. 10. It indicates that small changes in the thickness of the boundary layer can change the concentration in a magnetic fluid.

Fig. 11 indicates the effects of the chemical reaction parameter on the concentration profiles. The chemical reaction parameter $\gamma$ has a negligible effect on the velocity profile and heat transfer. Therefore, it is not plotted here. The velocity and temperature distributions remain the same, as shown in Figs. 8 and 9, for different values of $\gamma$. However, increasing the values of $\gamma$, the concentration in the flow decreases.

Fig. 12 represents the temperature distributions for different values of the heat generation/absorption parameter $(\varphi)$. Increasing the values of $\varphi$, there is no change in the velocity distribution. Therefore, it is not presented in the results. In the present problem, the parameter $\varphi$ works as the heat absorption parameter. As the values of $\varphi$ increases, the heat transfer decreases in the magnetic fluid. In Fig. 13, the effect of the absorption parameter on the concentration profile can be seen. If heat is absorbed during the flow, the concentration also decreases. For large values of $\varphi$, the concentration decreases at a linear rate.

\section{Conclusions}

A numerical solution is obtained through mathematical modelling. These results are very significant and reliable as compared to previously published results. The unsteady behaviour of MHD flow is also an important aspect of this study, which has been previously avoided by researchers due to its complexity. On detailed analysis, the physical parameters involved in the results might be applied in real-life applications on a priority basis. This kind of numerical approach through mathematical modelling of the problem has not been published yet.

\section{References}

[1] Das, S., Tarafdar, B., Jana, R.N. (2018). Hall effects on unsteady MHD rotating flow past a periodically accelerated porous plate with slippage. European Journal of Mechanics-B/Fluids, 72, 135-143.

[2] Tian, X.-Y., Li, B.-W., Zhang, J.-K. (2017). The effects of radiation optical properties on the unsteady 2D boundary layer MHD flow and heat transfer over a stretching plate. International Journal of Heat and Mass Transfer, 105, 109-123.

[3] Dogonchi, A.S., Ganji, D.D. (2017). Impact of Cattaneo-Christov heat flux on MHD nanofluid flow and heat transfer between parallel plates considering thermal radiation effect. Journal of the Taiwan Institute of Chemical Engineers, 80, 52-63.

[4] Makinde, O.D., Khan, W.A., Culham, J.R. (2016). MHD variable viscosity reacting flow over a convectively heated plate in a porous medium with thermophoresis and radiative heat transfer. International Journal of Heat and Mass Transfer, 93, 595-604.

[5] Raza, J., Rohni, A.M., Omar, Z. (2016). MHD flow and heat transfer of $\mathrm{Cu}$-water nanofluid in a semi porous channel with stretching walls. International Journal of Heat and Mass Transfer, 103, 336-340.

[6] Akl, M.Y. (2009). MHD flow and heat transfer driving by a power-law shear over a semi-infinite flat plate. Computational Materials Science, 45(2), 271-274.

[7] Ali, F., Gohar, M., Khan, I. (2016). MHD flow of water-based Brinkman type nanofluid over a vertical plate embedded in a porous medium with variable surface velocity, temperature and concentration. Journal of Molecular Liquids, 223, 412-419.

[8] Rout, P.K., Sahoo, S.N., Dash, G.C., Mishra, S.R. (2016). Chemical reaction effect on MHD free convection flow in a micropolar fluid. Alexandria Engineering Journal, 55(3), 29672973.

[9] Alam, M.S., Rahman, M.M., Sattar, M.A. (2008). Effects of variable suction and thermophoresis on steady MHD combined free-forced convective heat and mass transfer flow over a semiinfinite permeable inclined plate in the presence of thermal radiation. International Journal of Thermal Sciences, 47(6), 758-765.

[10] Rao, J.A., Shivaiah, S. (2011). Chemical reaction effects on unsteady MHD flow past semi-infinite vertical porous plate 
with viscous dissipation. Applied Mathematics and Mechanics, 32(8), 1065.

[11] Ibrahim, F.S., Elaiw, A.M., Bakr, A.A. (2008). Effect of the chemical reaction and radiation absorption on the unsteady MHD free convection flow past a semi infinite vertical permeable moving plate with heat source and suction. Communications in Nonlinear Science and Numerical Simulation, 13(6), 1056-1066.

[12] Alboussiere, T., Garandet, J.P., Moreau, R. (1996). Asymptotic analysis and symmetry in MHD convection. Physics of Fluids, 8(8), 2215-2226.

[13] VeeraKrishna, M., Chamkha, A.J. (2018). Hall effects on unsteady MHD flow of second grade fluid through porous medium with ramped wall temperature and ramped surface concentration. Physics of Fluids, 30(5), 053101.

[14] Bluck, M.J., Wolfendale, M.J. (2015). An analytical solution to electromagnetically coupled duct flow in MHD. Journal of Fluid Mechanics, 771, 595-623.

[15] Seth, G.S., Sarkar, S. (2015). MHD natural convection heat and mass transfer flow past a time dependent moving vertical plate with ramped temperature in a rotating medium with Hall effects, radiation and chemical reaction. Journal of Mechanics, 31(1), 91-104.

[16] Kumar, R., Raju, C.S.K., Sekhar, K.R., Reddy, G.V. (2017). Three dimensional MHD ferrous nanofluid flow over a sheet of variable thickness in slip flow regime. Journal of Mechanics, 1-12

[17] Berton, R.P.H. (2018). Analytic model of a resistive magnetohydrodynamic shock without Hall effect. Journal of Fluid Mechanics, 842, 273-322.

[18] Briard, A., Gomez, T. (2018). The decay of isotropic magnetohydrodynamics turbulence and the effects of crosshelicity. Journal of Plasma Physics, 84(1).

[19] Borisevich, V.D., Potanin, E.P., Whichello, J. (2017). Circulation control in magnetohydrodynamic rotating flows. Journal of Fluid Mechanics, 829, 328-344.

[20] Hayat, T., Rashid, M., Imtiaz, M., Alsaedi, A. (2017). Nanofluid flow due to rotating disk with variable thickness and homogeneous-heterogeneous reactions. International Journal of Heat and Mass Transfer, 113, 96-105.

[21] Hayat, T., Rashid, M., Imtiaz, M., Alsaedi, A. (2017). MHD convective flow due to a curved surface with thermal radiation and chemical reaction. Journal of Molecular Liquids, 225, 482489.

[22] Hayat, T., Rashid, M., Imtiaz, M., Alsaedi, A. (2017). MHD effects on a thermo-solutal stratified nanofluid flow on an exponentially radiating stretching sheet. Journal of Applied Mechanics and Technical Physics 58(2), 214-223.

[23] Hayat, T. et al. (2015). Magnetohydrodynamic (MHD) stretched flow of nanofluid with power-law velocity and chemical reaction. AIP Advances, 5 (11), 117121. 